\title{
Linearized Riccati Technique and (Non-)Oscillation Criteria for Half-Linear Difference Equations
}

\author{
Ondřej Došlý1 and Simona Fišnarová ${ }^{2}$ \\ ${ }^{1}$ Department of Mathematics and Statistics, Masaryk University, Janáčkovo nám. 2a, \\ 66295 Brno, Czech Republic \\ ${ }^{2}$ Department of Mathematics, Mendel University of Agriculture and Forestry in Brno, Zemědělská 1, \\ 61300 Brno, Czech Republic
}

Correspondence should be addressed to Ondřej Došlý, dosly@math.muni.cz

Received 23 August 2007; Accepted 26 November 2007

Recommended by John R. Graef

We consider the half-linear second-order difference equation $\Delta\left(r_{k} \Phi\left(\Delta x_{k}\right)\right)+c_{k} \Phi\left(x_{k+1}\right)=0, \Phi(x):=$ $|x|^{p-2} x, p>1$, where $r, c$ are real-valued sequences. We associate with the above-mentioned equation a linear second-order difference equation and we show that oscillatory properties of the abovementioned one can be investigated using properties of this associated linear equation. The main tool we use is a linearization technique applied to a certain Riccati-type difference equation corresponding to the above-mentioned one.

Copyright ( $) 2008$ O. Došlý and S. Fišnarová. This is an open access article distributed under the Creative Commons Attribution License, which permits unrestricted use, distribution, and reproduction in any medium, provided the original work is properly cited.

\section{Introduction}

In this paper, we deal with oscillatory properties of solutions of the half-linear second-order difference equation

$$
\Delta\left(r_{k} \Phi\left(\Delta x_{k}\right)\right)+c_{k} \Phi\left(x_{k+1}\right)=0, \quad \Phi(x):=|x|^{p-2} x, \quad p>1
$$

where $r, c$ are real-valued sequences and $r_{k}>0$. This equation can be regarded as a discrete counterpart of the half-linear differential equation

$$
\left(r(t) \Phi\left(x^{\prime}\right)\right)^{\prime}+c(t) \Phi(x)=0
$$

which attracted considerable attention in the recent years. We refer to the books in [1, 2] and the references given therein. The basic qualitative theory of (1.1) has been established in the series of papers in [3-7] and it is summarized in the books [8, Chapter 3] and [2, Chapter 8]. 
It is known that oscillatory properties of (1.1) are very similar to those of the second-order Sturm-Liouville difference equation (which is a special case of $p=2$ in (1.1)):

$$
\Delta\left(r_{k} \Delta x_{k}\right)+c_{k} x_{k+1}=0
$$

In particular, the discrete linear Sturmian theory extends verbatim to (1.1), and hence this equation can be classified as oscillatory or nonoscillatory. We will recall elements of the oscillation theory of (1.1) in more detail in the next section.

The basic idea of the discrete linearization technique which we establish in this paper is motivated by the paper of Elbert and Schneider [9], where the second-order half-linear differential equation

$$
\left(\Phi\left(x^{\prime}\right)\right)^{\prime}+\frac{\gamma_{p}}{t^{p}} \Phi(x)+2\left(\frac{p-1}{p}\right)^{p-1} \frac{\delta(t)}{t^{p}} \Phi(x)=0, \quad \gamma_{p}:=\left(\frac{p-1}{p}\right)^{p},
$$

is viewed as a perturbation of the Euler-type half-linear differential equation

$$
\left(\Phi\left(x^{\prime}\right)\right)^{\prime}+\frac{\gamma_{p}}{t^{p}} \Phi(x)=0
$$

and oscillatory properties of (1.4) are studied via the linear equation

$$
\left(t y^{\prime}\right)^{\prime}+\frac{\delta(t)}{t} y=0
$$

under the assumption that $\int_{t}^{\infty} \delta(s) / s d s \geq 0$ for large $t$. In particular, the following statements are presented in [9].

(i) Let $p \geq 2$ and let linear equation (1.6) be nonoscillatory. Then (1.4) is also nonoscillatory.

(ii) Let $p \in(1,2]$ and let half-linear equation (1.4) be nonoscillatory. Then linear equation (1.6) is also nonoscillatory.

The linearization technique for (1.2) has been further developed in [10-12]; see also references given therein.

In our paper, we introduce a similar linearization technique for the investigation of oscillatory properties of (1.1). This equation is regarded as a perturbation of the nonoscillatory equation of the same form:

$$
\Delta\left(r_{k} \Phi\left(\Delta x_{k}\right)\right)+\tilde{c}_{k} \Phi\left(x_{k+1}\right)=0
$$

and oscillatory properties of solutions of (1.1) are related to those of the linear second-order difference equation

$$
\Delta\left(R_{k} \Delta y_{k}\right)+C_{k} y_{k+1}=0
$$

where

$$
R_{k}=\frac{2}{q} r_{k} h_{k} h_{k+1}\left|\Delta h_{k}\right|^{p-2}, \quad C_{k}=\left(c_{k}-\widetilde{c}_{k}\right) h_{k+1}^{p}
$$


with $q=p /(p-1)$ being the conjugate number of $p$, and with a certain distinguished solution $h$ of (1.7). This enables to apply the deeply developed linear oscillation theory when investigating oscillations of half-linear equation (1.1). As we will see in the next sections, compared to the continuous case, the linearization technique is technically more difficult in the discrete case since a nonlinear function which appears in the so-called modified Riccati equation is considerably more complicated in the discrete case.

The paper is organized as follows. In the next section, we recall basic oscillatory properties of (1.1), including a quadratization formula for a certain nonlinear function which plays an important role in subsequent sections of the paper. In Section 3, we present a discrete version of the above-mentioned result of Elbert and Schneider [9]. In Section 4, we show that under certain additional restriction on properties of solutions of (1.7) we do not need to distinguish between the cases $p \geq 2$ and $p \in(1,2]$. The last section of the paper is devoted to an application of the results of the previous sections of the paper.

\section{Preliminaries}

Oscillatory properties of (1.1) are defined using the concept of the generalized zero which is defined in the same way as for (1.3) (see, e.g., [8, Chapter 3] or [2, Chapter 7]). A solution $x$ of (1.1) has a generalized zero in an interval $(m, m+1]$ if $x_{m} \neq 0$ and $x_{m} x_{m+1} r_{m} \leq 0$. Since we suppose that $r_{k}>0$ (oscillation theory of (1.1) generally requires only $r_{k} \neq 0$ ), a generalized zero of $x$ in $(m, m+1]$ is either a "real" zero at $k=m+1$ or the sign change between $m$ and $m+1$. Equation (1.1) is said to be disconjugate in a discrete interval $[m, n]$ if the solution $x$ of (1.1) given by the initial condition $x_{m}=0, x_{m+1} \neq 0$, has no generalized zero in $(m, n+1]$. Equation (1.1) is said to be nonoscillatory if there exists $m \in \mathbb{N}$ such that it is disconjugate on $[m, n]$ for every $n>m$, and it is said to be oscillatory in the opposite case.

If $x$ is a solution of (1.1) such that $x_{k} \neq 0$ in some discrete interval $[m, \infty)$, then $w_{k}=$ $r_{k} \Phi\left(\Delta x_{k} / x_{k}\right)$ is a solution of the associated Riccati-type equation

$$
R\left[w_{k}\right]:=\Delta w_{k}+c_{k}+w_{k}\left(1-\frac{r_{k}}{\Phi\left(\Phi^{-1}\left(r_{k}\right)+\Phi^{-1}\left(w_{k}\right)\right)}\right)=0
$$

Moreover, if $x$ has no generalized zero in $[m, \infty)$, then $\Phi^{-1}\left(r_{k}\right)+\Phi^{-1}\left(w_{k}\right)>0, k \in[m, \infty)$. If we suppose that (1.1) is nonoscillatory, among all solutions of (2.1) there exists the so-called distinguished solution $\widetilde{w}$ which has the property that there exists an interval $[m, \infty)$ such that any other solution $w$ of (2.1) for which $\Phi^{-1}\left(r_{k}\right)+\Phi^{-1}\left(w_{k}\right)>0, k \in[m, \infty)$, satisfies $w_{k}>\widetilde{w}_{k}$, $k \in[m, \infty)$. Therefore, the distinguished solution of (2.1) is, in a certain sense, minimal solution of this equation near $\infty$. If $\widetilde{w}$ is the distinguished solution of (2.1), then the associated solution of (1.1) given by the formula

$$
\tilde{x}_{k}=\prod_{j=m}^{k-1}\left[1+\Phi^{-1}\left(\frac{\tilde{w}_{j}}{r_{j}}\right)\right]
$$

is said to be the recessive solution of (1.1) (see [13]). Note that in the linear case $p=2$ a solution $\tilde{x}$ of (1.3) is recessive if and only if

$$
\sum^{\infty} \frac{1}{r_{k} \tilde{x}_{k} \tilde{x}_{k+1}}=\infty
$$


Our first statement presents a comparison theorem for distinguished solutions of (2.1) and (2.4) given below.

Lemma 2.1 (see [13]). Let (1.1) be nonoscillatory and let $c_{k} \geq \widetilde{c}_{k}$ for large $k$. Further, let $\widetilde{w}_{k}, \widetilde{v}_{k}$ be distinguished solutions of the corresponding generalized Riccati equations (2.1) and

$$
\tilde{R}\left[v_{k}\right]:=\Delta v_{k}+\tilde{c}_{k}+v_{k}\left(1-\frac{r_{k}}{\Phi\left(\Phi^{-1}\left(r_{k}\right)+\Phi^{-1}\left(v_{k}\right)\right)}\right)=0
$$

respectively. Then there exists $m \in \mathbb{Z}$ such that $\tilde{\boldsymbol{w}}_{k} \geq \tilde{\boldsymbol{v}}_{k}$ for $k \in[m, \infty)$. In particular, if $c_{k} \geq 0$ and $\Sigma^{\infty} r_{k}^{1-q}=\infty$, then $\tilde{w}_{k} \geq 0$ for large $k$.

The next statement relates nonoscillation of (1.1) to the existence of a certain solution of the Riccati inequality associated with (2.1).

Lemma 2.2 (see [2, Theorem 8.2.7]). Equation (1.1) is nonoscillatory if and only if there exists a sequence $w_{k}$ satisfying $r_{k}+w_{k}>0$ and

$$
R\left[w_{k}\right] \leq 0
$$

for large $k$.

The next statement is the discrete version of the generalized Leighton-Wintner oscillation criterion. In this criterion, (1.1) is viewed as a perturbation of (1.7).

Lemma 2.3 (see [13]). Let $h$ be the positive recessive solution of nonoscillatory equation (1.7). If

$$
\sum^{\infty}\left(c_{k}-\widetilde{c}_{k}\right) h_{k+1}^{p}=\infty
$$

then (1.1) is oscillatory.

The last auxiliary oscillation results of this section are Hille-Nehari (non-)oscillation criteria for linear difference equation (1.3).

Lemma 2.4 (see [14]). Suppose that $c_{k} \geq 0, r_{k}>0, \Sigma^{\infty} r_{k}^{-1}=\infty$, and $\sum^{\infty} c_{k}<\infty$. If

$$
\liminf _{k \rightarrow \infty}\left(\sum^{k-1} \frac{1}{r_{j}}\right)\left(\sum_{j=k}^{\infty} c_{j}\right)>\frac{1}{4},
$$

then (1.3) is oscillatory. If

$$
\limsup _{k \rightarrow \infty}\left(\sum^{k-1} \frac{1}{r_{j}}\right)\left(\sum_{j=k}^{\infty} c_{j}\right)<\frac{1}{4},
$$

then (1.3) is nonoscillatory. 
For the remaining part of this section, we suppose that (1.7) is nonoscillatory and we let $h$ be its solution such that $h_{k}>0$ for large $k$. Further, put

$$
G_{k}:=r_{k} h_{k} \Phi\left(\Delta h_{k}\right)
$$

and define the function

$$
H(k, v):=v+r_{k} h_{k+1} \Phi\left(\Delta h_{k}\right)-\frac{r_{k}\left(v+G_{k}\right) h_{k+1}^{p}}{\Phi\left(h_{k}^{q} \Phi^{-1}\left(r_{k}\right)+\Phi^{-1}\left(v+G_{k}\right)\right)}
$$

Lemma 2.5. Put

$$
v_{k}:=h_{k}^{p}\left(w_{k}-\widetilde{w}_{k}\right)
$$

where $\tilde{w}_{k}=r_{k} \Phi\left(\Delta h_{k} / h_{k}\right)$ is a solution of (2.4) and $w_{k}$ is any sequence satisfying $r_{k}+w_{k} \neq 0$. Then

$$
\Delta v_{k}+\left(c_{k}-\widetilde{c}_{k}\right) h_{k+1}^{p}+H\left(k, v_{k}\right)=h_{k+1}^{p} R\left[w_{k}\right] .
$$

In particular, if $w_{k}$ is a solution of (2.1), then

$$
\Delta v_{k}+\left(c_{k}-\tilde{c}_{k}\right) h_{k+1}^{p}+H\left(k, v_{k}\right)=0
$$

Moreover, $H(k, v) \geq 0$ for $v>-r_{k} h_{k}\left(\Phi\left(\Delta h_{k}\right)+h_{k}^{p-1}\right)$ with the equality if and only if $v=0$.

Proof. By a direct computation and using the fact that $\widetilde{w}_{k}$ is a solution of (2.4), we obtain

$$
\begin{aligned}
\Delta v_{k} & =h_{k+1}^{p}\left(w_{k+1}-\widetilde{w}_{k+1}\right)-v_{k} \\
& =h_{k+1}^{p}\left(w_{k+1}+\tilde{c}_{k}-\frac{r_{k} \tilde{w}_{k}}{\Phi\left(\Phi^{-1}\left(r_{k}\right)+\Phi^{-1}\left(\tilde{w}_{k}\right)\right)}\right)-v_{k} \\
& =h_{k+1}^{p}\left(w_{k+1}+\tilde{c}_{k}-r_{k} \Phi\left(\frac{\Delta h_{k}}{h_{k+1}}\right)\right)-v_{k} \\
& =h_{k+1}^{p}\left(w_{k+1}+\widetilde{c}_{k}\right)-r_{k} h_{k+1} \Phi\left(\Delta h_{k}\right)-v_{k} .
\end{aligned}
$$

Next, since $v_{k}=h_{k}^{p} w_{k}-G_{k}$, we have

$$
\begin{aligned}
\frac{r_{k}\left(v_{k}+G_{k}\right)}{\Phi\left(h_{k}^{q} \Phi^{-1}\left(r_{k}\right)+\Phi^{-1}\left(v_{k}+G_{k}\right)\right)} & =\frac{r_{k} h_{k}^{p} w_{k}}{\Phi\left(h_{k}^{q} \Phi^{-1}\left(r_{k}\right)+\Phi^{-1}\left(h_{k}^{p} w_{k}\right)\right)} \\
& =\frac{r_{k} w_{k}}{\Phi\left(\Phi^{-1}\left(r_{k}\right)+\Phi^{-1}\left(w_{k}\right)\right)}
\end{aligned}
$$

and hence

$$
\begin{aligned}
\Delta v_{k}+\left(c_{k}-\tilde{c}_{k}\right) h_{k+1}^{p}+H\left(k, v_{k}\right) & =h_{k+1}^{p}\left(w_{k+1}+c_{k}-\frac{r_{k}\left(v_{k}+G_{k}\right)}{\Phi\left(h_{k}^{q} \Phi^{-1}\left(r_{k}\right)+\Phi^{-1}\left(v_{k}+G_{k}\right)\right)}\right) \\
& =h_{k+1}^{p} R\left[w_{k}\right] .
\end{aligned}
$$


If $w_{k}$ is a solution of (2.1), then $v_{k}$ satisfies (2.13). We prove the nonnegativity of the function $H(k, v)$ for $v>-r_{k} h_{k}\left(\Phi\left(\Delta h_{k}\right)+h_{k}^{p-1}\right)$ as follows. By a direct computation, we have

$$
\begin{aligned}
& H_{v}(k, v)=1-\frac{r_{k}^{q} h_{k}^{q} h_{k+1}^{p}}{\left(h_{k}^{q} \Phi^{-1}\left(r_{k}\right)+\Phi^{-1}\left(v_{k}+G_{k}\right)\right)^{p}} \\
& H_{v v}(k, v)=\frac{q r_{k}^{q} h_{k}^{q} h_{k+1}^{p}\left|v_{k}+G_{k}\right|^{q-2}}{\left(h_{k}^{q} \Phi^{-1}\left(r_{k}\right)+\Phi^{-1}\left(v_{k}+G_{k}\right)\right)^{p+1}} .
\end{aligned}
$$

Hence $H_{v}(k, v)=0$ if and only if $v=0$ and the function $H(k, v)$ is convex with respect to $v$ for $v$ satisfying $h_{k}^{-q} \Phi^{-1}\left(r_{k}\right)+\Phi^{-1}\left(v+G_{k}\right)>0$ which is equivalent to $v>-r_{k} h_{k}\left(\Phi\left(\Delta h_{k}\right)+h_{k}^{p-1}\right)$. This proves the last statement of Lemma 2.5.

Lemma 2.6. Let $R, G$ be defined by (1.9) and (2.9), respectively, and suppose that $G_{k}>0$ for $k \in \mathbb{N}$. Then we have the following inequalities for $v \geq 0$ and $k \in \mathbb{N}$ :

$$
\begin{array}{ll}
\left(R_{k}+v\right) H(k, v) \geq v^{2}, & p \in(1,2], \\
\left(R_{k}+v\right) H(k, v) \leq v^{2}, & p \geq 2 .
\end{array}
$$

Proof. In this proof, we write explicitly an index by a sequence only if this index is different from $k$; that is, no index means the index $k$. In addition to (2.17), we have

$$
H_{v v v}(k, 0)=\frac{q}{r^{2} h^{2} h_{k+1}^{2}(\Delta h)^{2 p-3}}\left[(q-2) h_{k+1}-(2 q-1) \Delta h\right] .
$$

Denote $F(k, v):=\left(R_{k}+v\right) H(k, v)-v^{2}$. Then we have $F_{v}(k, 0)=0=F_{v v}(k, 0)$ and

$$
\begin{aligned}
F_{v v v}(k, 0) & =R H_{v v v}(k, 0)+3 H_{v v}(k, 0) \\
& =\frac{2}{r h h_{k+1}(\Delta h)^{p-1}}\left[(q-2) h_{k+1}-(2 q-1) \Delta h\right]+\frac{3 q}{r h h_{k+1}(\Delta h)^{p-2}} \\
& =\frac{1}{r h h_{k+1}(\Delta h)^{p-1}}[2(q-2)(h+\Delta h)+(2-q) \Delta h] \\
& =\frac{q-2}{r h h_{k+1}(\Delta h)^{p-1}}[h+h+\Delta h]=\frac{q-2}{r h h_{k+1}(\Delta h)^{p-1}}\left[h+h_{k+1}\right] .
\end{aligned}
$$

Consequently,

$$
\operatorname{sgn} F(k, v)=\operatorname{sgn}(q-2)
$$

in some right neighborhood of $v=0$. Further, we have

$$
\begin{aligned}
F_{v v}(k, v) & =2 H_{v}(k, v)+(R+v) H_{v v}(k, v)-2 \\
& =-\frac{2 r^{q} h^{q} h_{k+1}^{p}}{\left(h^{q} \Phi^{-1}(r)+\Phi^{-1}(v+G)\right)^{p}}+\frac{q r^{q} h^{q} h_{k+1}^{p}(v+G)^{q-2}(R+v)}{\left(h^{q} \Phi^{-1}(r)+\Phi^{-1}(v+G)\right)^{p+1}} \\
& =\frac{r^{q} h^{q} h_{k+1}^{p}}{\left(h^{q} \Phi^{-1}(r)+\Phi^{-1}(v+G)\right)^{p+1}}\left[-2 r^{q-1} h^{q}-2 \Phi^{-1}(v+G)+q(v+G)^{q-2}(R+v)\right] .
\end{aligned}
$$


Denote by $A(v)$ the expression in brackets in the last expression. By a direct computation, we have

$$
A(v)=(q-2) \Phi^{-1}(v+G)+q(R-G)(v+G)^{q-2}-2 r^{q-1} h^{q}
$$

hence sgn $A(v)=\operatorname{sgn} F_{v v}(k, v)=\operatorname{sgn}(q-2)$ for large $v$, and from the computation of $F_{v v v}(k, 0)$, we also have $(q-2) A(v)>0$ in some right neighborhood of $v=0$. Since

$$
A^{\prime}(v)=(q-2)(v+G)^{q-3}[(q-1)(v+G)+q(R-G)]
$$

has no positive root (observe that $(q-1)(v+G)+q(R-G)=0$ if and only if $v=$ $\left.-(1 /(q-1)) r h(\Delta h)^{p-2}\left(h_{k+1}+h\right)<0\right)$, this means that $(q-2) A(v)$ and hence also $(q-2) F_{v v}(k, v)$ have a constant sign for $v \in(0, \infty)$. Therefore, the function $F(k, v)$ is convex for $q \geq 2$ and concave for $q \leq 2$, and this together with (2.21) implies the required inequalities.

\section{3. (Non-)oscillation criteria: $p \geq 2$ versus $p \in(1,2]$}

In this section, we suppose that (1.7) is nonoscillatory and possesses a positive increasing solution $h$. We associate with (1.1) the linear Sturm-Liouville second-order difference equation

$$
\Delta\left(R_{k} \Delta y_{k}\right)+C_{k} y_{k+1}=0
$$

where $R$ and $C$ are given by (1.9), that is,

$$
R_{k}=\frac{2}{q} r_{k} h_{k} h_{k+1}\left(\Delta h_{k}\right)^{p-2}, \quad C_{k}=\left(c_{k}-\widetilde{c}_{k}\right) h_{k+1}^{p} .
$$

The results of this section can be regarded as a discrete version of the results given in [9].

Theorem 3.1. Let $p \geq 2, c_{k} \geq \widetilde{c}_{k}$ for large $k$,

$$
\sum^{\infty} \frac{1}{R_{k}}=\infty,
$$

and suppose that linear equation (3.1) with $R, C$ given by (1.9) is nonoscillatory. Then half-linear equation (1.1) is also nonoscillatory.

Proof. The proof is based on Lemma 2.2. Nonoscillation of (3.1) implies the existence of a solution $v$ of the associated Riccati equation

$$
\Delta v_{k}+C_{k}+\frac{v_{k}^{2}}{R_{k}+v_{k}}=0
$$

such that $R_{k}+v_{k}>0$ for large $k$. Moreover, since (3.3) holds and $C_{k} \geq 0$ for large $k$, by Lemma $2.1 v_{k} \geq 0$ for large $k$. By Lemma 2.6, we have $\left(R_{k}+v\right) H(k, v) \leq v^{2}$; hence $v$ is also a solution of the inequality

$$
\Delta v_{k}+C_{k}+H\left(k, v_{k}\right) \leq 0 .
$$

Now, substituting for $v=h^{p}(w-\widetilde{w})$, where $\widetilde{w}=r \Phi(\Delta h / h)$, we see from Lemma 2.5 that $w$ is a solution of Riccati inequality (2.5). Moreover, $r_{k}+w_{k}=r_{k}+h_{k}^{-p} v_{k}+\tilde{w}_{k}>0$ since $v_{k} \geq 0$ and $h$ is a nonoscillatory solution of (1.7); that is, the corresponding solution of the associated Riccati equation $\tilde{w}$ satisfies $r_{k}+\widetilde{w}_{k}>0$. Therefore, (1.1) is nonoscillatory. 
Theorem 3.2. Let $p \in(1,2], c_{k} \geq \widetilde{c}_{k}$ for large $k$, and let $h$ be the recessive solution of (1.7). If halflinear equation (1.1) is nonoscillatory, then linear equation (3.1) is also nonoscillatory.

Proof. We proceed similarly as in the previous proof. Nonoscillation of (1.1) implies the existence of the distinguished solution $w$ of the associated Riccati equation (2.1) such that $w_{k}+r_{k}>0$ for large $k$. Put again $v=h^{p}(w-\tilde{w})$, where $\widetilde{w}$ is the distinguished solution of (2.4). Then $v$ solves the equation

$$
\Delta v_{k}+C_{k}+H\left(k, v_{k}\right)=0
$$

and by Lemma 2.1, we have $w_{k} \geq \tilde{w}_{k}$ for large $k$, hence $v_{k} \geq 0$, and therefore $R_{k}+v_{k}>0$ for large $k$. By Lemma 2.6,

$$
\Delta v_{k}+C_{k}+\frac{v_{k}^{2}}{R_{k}+v_{k}} \leq 0
$$

This means that (3.1) is nonoscillatory by Lemma 2.2.

\section{Criteria without restriction on $p$}

Throughout this section, we suppose that $R_{k}, C_{k}$, and $G_{k}$ are given by (1.9) and (2.9), respectively, and that (1.7) is nonoscillatory.

Theorem 4.1. Let $c_{k} \geq \widetilde{c}_{k}$ for large $k$ and let $h_{k}>0$ be the recessive solution of (1.7) such that

$$
\sum^{\infty}\left(c_{k}-\widetilde{c}_{k}\right) h_{k+1}^{p}<\infty
$$

Further, suppose that condition (3.3) holds and

$$
\lim _{k \rightarrow \infty} r_{k} h_{k} \Phi\left(\Delta h_{k}\right)=\infty
$$

If there exists $\varepsilon>0$ such that the equation

$$
\Delta\left(R_{k} \Delta y_{k}\right)+(1-\varepsilon) C_{k} y_{k+1}=0
$$

is oscillatory, then (1.1) is also oscillatory.

Proof. Let $\varepsilon>0$ be such that (4.3) is oscillatory (i.e., $\varepsilon<1)$. Suppose, by contradiction, that (1.1) is nonoscillatory, and let $x_{k}$ be its recessive solution. Denote by $w_{k}=r_{k} \Phi\left(\Delta x_{k} /\right.$ $\left.x_{k}\right)$ and $\tilde{w}_{k}=r_{k} \Phi\left(\Delta h_{k} / h_{k}\right)$ the distinguished solutions of the Riccati equations (2.1) and (2.4), respectively, and put $v_{k}:=h_{k}^{p}\left(w_{k}-\widetilde{w}_{k}\right)$. Since $c_{k} \geq \widetilde{c}_{k}$ for large $k$, it follows from Lemma 2.1 that $w_{k} \geq \widetilde{w}_{k}$, and hence also $v_{k} \geq 0$ for large $k$. According to Lemma 2.5, we have

$$
\Delta v_{k}=-C_{k}-H\left(k, v_{k}\right)
$$

Hence $v_{k}$ is nonnegative and nonincreasing for large $k$, and this means that there exists a limit of $v_{k}$ such that

$$
0 \leq \lim _{k \rightarrow \infty} v_{k}<\infty
$$


Next, let $N \in \mathbb{N}$ be sufficiently large, $k>N$. Summing (4.4) from $N$ to $k$, we obtain

$$
v_{N}-v_{k+1}=\sum_{j=N}^{k} C_{j}+\sum_{j=N}^{k} H\left(j, v_{j}\right),
$$

and hence

$$
v_{N} \geq \sum_{j=N}^{k} C_{j}+\sum_{j=N}^{k} H\left(j, v_{j}\right)
$$

Letting $k \rightarrow \infty$ and using condition (4.1), we have

$$
\sum^{\infty} H\left(k, v_{k}\right)<\infty
$$

Substituting $z_{k}=v_{k} / G_{k}$ into $H\left(k, v_{k}\right)$, we obtain

$$
H\left(k, G_{k} z_{k}\right)=G_{k} z_{k}+r_{k} h_{k+1} \Phi\left(\Delta h_{k}\right)-\frac{r_{k}\left(z_{k}+1\right) h_{k+1}^{p}}{\Phi\left(h_{k} / \Delta h_{k}+\Phi^{-1}\left(z_{k}+1\right)\right)}=: \widetilde{H}\left(k, z_{k}\right) .
$$

Now, it follows from conditions (4.2) and (4.5) that $z_{k} \rightarrow 0$ as $k \rightarrow \infty$. Hence we may approximate the function $\widetilde{H}(k, z)$ by the second-degree Taylor polynomial at the center $z=0(k$ is regarded as a parameter). By a direct computation, we have

$$
\widetilde{H}(k, 0)=0, \quad \widetilde{H}_{z}(k, 0)=0, \quad \widetilde{H}_{z z}(k, 0)=\frac{q r_{k} h_{k}\left(\Delta h_{k}\right)^{p}}{h_{k+1}},
$$

and hence

$$
\widetilde{H}(k, z)=\frac{q r_{k} h_{k}\left(\Delta h_{k}\right)^{p}}{2 h_{k+1}} z^{2}+o\left(z^{2}\right) \quad \text { as } z \longrightarrow 0
$$

The term $o\left(z^{2}\right)$ is of the form $\widetilde{H}_{z z z}(k, \xi) z^{3}$ for some $\xi \in(0, z)$. By a direct computation, we have

$$
\widetilde{H}_{z z z}(k, 0)=q r_{k} h_{k} \frac{\left(\Delta h_{k}\right)^{p}}{h_{k+1}^{2}}\left[(q-2) h_{k+1}-(2 q-1) \Delta h_{k}\right],
$$

that is,

$$
\left|\widetilde{H}_{z z z}(k, 0)\right| \leq q r_{k} h_{k} \frac{\left(\Delta h_{k}\right)^{p}}{h_{k+1}}[|q-2|+(2 q-1)]
$$

Since $\widetilde{H}_{z z z}(k, z)$ is continuous with respect to $z$ near $z=0$, there exists a constant $M>0$ such that

$$
\left|\widetilde{H}_{z z z}(k, \xi)\right| \leq M r_{k} h_{k} \frac{\left(\Delta h_{k}\right)^{p}}{h_{k+1}}
$$


and hence (4.11) can be written in the form

$$
\widetilde{H}(k, z)=q r_{k} h_{k} \frac{\left(\Delta h_{k}\right)^{p}}{2 h_{k+1}} z^{2}(1+o(1)) \quad \text { as } z \longrightarrow 0
$$

and the convergence $o(1) \rightarrow 0$ as $z \rightarrow 0$ is uniform with respect to $k$. This means that there exists $N_{1}$ such that

$$
\frac{(q-\varepsilon) r_{k} h_{k}\left(\Delta h_{k}\right)^{p}}{2 h_{k+1}} z_{k}^{2}<\widetilde{H}\left(k, z_{k}\right)<\frac{(q+\varepsilon) r_{k} h_{k}\left(\Delta h_{k}\right)^{p}}{2 h_{k+1}} z_{k}^{2} \quad \text { for } k \geq N_{1},
$$

and consequently

$$
\begin{aligned}
\infty & >\sum^{\infty} H\left(k, v_{k}\right)=\sum^{\infty} \widetilde{H}\left(k, z_{k}\right)>\frac{q-\varepsilon}{2} \sum^{\infty} \frac{r_{k} h_{k}\left(\Delta h_{k}\right)^{p}}{h_{k+1}} z_{k}^{2} \\
& =\frac{q-\varepsilon}{2} \sum^{\infty} \frac{r_{k} h_{k}\left(\Delta h_{k}\right)^{p} v_{k}^{2}}{h_{k+1} G_{k}^{2}}=\frac{q-\varepsilon}{2} \sum^{\infty} \frac{v_{k}^{2}}{r_{k} h_{k} h_{k+1}\left(\Delta h_{k}\right)^{p-2}} .
\end{aligned}
$$

Taking into account condition (3.3), it follows that $v_{k} \rightarrow 0$ as $k \rightarrow \infty$. Thus we can apply Taylor's formula to the function $\widetilde{F}(k, v):=\left(R_{k}+v\right) H(k, v)$ at the center $v=0$. By a direct computation (see also the proof of Lemma 2.6), we have ( $k$ is regarded again as a parameter)

$$
\widetilde{F}(k, 0)=0, \quad \tilde{F}_{v}(k, 0)=0, \quad \tilde{F}_{v v}(k, 0)=2,
$$

and hence

$$
\tilde{F}(k, v)=v^{2}+o\left(v^{2}\right)=v^{2}(1+o(1)) \quad \text { as } v \longrightarrow 0 .
$$

Similarly as in the case of $\widetilde{H}(k, z)$, the convergence $o(1) \rightarrow 0$ as $v \rightarrow 0$ is uniform with respect to $k$ because of (4.2) and (2.20). Hence

$$
H(k, v)=\frac{v^{2}}{R_{k}+v}(1+o(1)) \quad \text { as } v \longrightarrow 0 .
$$

Consequently, there exists $N_{2}>N_{1}$ such that

$$
\left(1-\frac{\varepsilon}{2}\right) \frac{v_{k}^{2}}{R_{k}+v_{k}}<H\left(k, v_{k}\right)<\left(1+\frac{\varepsilon}{2}\right) \frac{v_{k}^{2}}{R_{k}+v_{k}} \quad \text { for } k \geq N_{2}
$$

Since

$$
R_{k}=\frac{2}{q} G_{k} \frac{h_{k+1}}{\Delta h_{k}}=\frac{2}{q} G_{k}\left(1+\frac{h_{k}}{\Delta h_{k}}\right)>\frac{2}{q} G_{k}
$$

from conditions (4.2) and (4.22) we have

$$
\frac{v_{k}}{R_{k}} \longrightarrow 0 \quad \text { as } k \longrightarrow \infty
$$


This means that there exists $N_{3} \in \mathbb{N}$ such that

$$
\frac{1-\varepsilon}{1+(1-\varepsilon)\left(v_{k} / R_{k}\right)}<\frac{1-\varepsilon / 2}{1+v_{k} / R_{k}} \text { for } k \geq N_{3}
$$

that is,

$$
\frac{1-\varepsilon}{R_{k}+(1-\varepsilon) v_{k}}=\frac{1}{R_{k} /(1-\varepsilon)+v_{k}}<\frac{1-\varepsilon / 2}{R_{k}+v_{k}} \quad \text { for } k \geq N_{3}
$$

Consequently, from (4.21) we obtain

$$
\frac{v_{k}^{2}}{R_{k} /(1-\varepsilon)+v_{k}}<H\left(k, v_{k}\right) \quad \text { for } k \geq \max \left\{N_{2}, N_{3}\right\},
$$

and according to Lemma 2.5,

$$
\Delta v_{k}+C_{k}+\frac{v_{k}^{2}}{R_{k} /(1-\varepsilon)+v_{k}}<0 \quad \text { for } k \geq \max \left\{N_{2}, N_{3}\right\} .
$$

The last inequality is the Riccati inequality associated with the equation

$$
\Delta\left(\frac{R_{k}}{1-\varepsilon} \Delta y_{k}\right)+C_{k} y_{k+1}=0
$$

that is, with (4.3). Since $R_{k} /(1-\varepsilon)+v_{k}>0$, it follows from Lemma 2.2 that this equation is nonoscillatory, which is a contradiction.

Theorem 4.2. Let $c_{k} \geq \widetilde{c}_{k}$ for large $k$ and let $h_{k}>0$ be a solution of (1.7) such that conditions (3.3), (4.1), and

$$
\liminf _{k \rightarrow \infty} r_{k} h_{k} \Phi\left(\Delta h_{k}\right)>0
$$

are satisfied. If there exists $\varepsilon>0$ such that the equation

$$
\Delta\left(R_{k} \Delta y_{k}\right)+(1+\varepsilon) C_{k} y_{k+1}=0
$$

is nonoscillatory, then (1.1) is also nonoscillatory.

Proof. It follows from nonoscillation of (4.30), that is,

$$
\Delta\left(\frac{R_{k}}{1+\varepsilon} \Delta y_{k}\right)+C_{k} y_{k+1}=0
$$

that there exists a solution $v_{k}$ of the associated Riccati equation

$$
\Delta v_{k}+C_{k}+\frac{v_{k}^{2}}{R_{k} /(1+\varepsilon)+v_{k}}=0
$$


such that $R_{k} /(1+\varepsilon)+v_{k}>0$. This means that $v_{k}$ is nonincreasing since $C_{k} \geq 0$ for large $k$. Moreover, since $\Sigma^{\infty}\left(1 / R_{k}\right)=\infty$, it follows from Lemma 2.1 that $v_{k} \geq 0$. Hence condition (4.5) holds. Summing (4.32) from $N$ to $k$ (let $N \in \mathbb{N}$ be sufficiently large, $k>N$ ), we obtain

$$
v_{N}-v_{k+1}=\sum_{j=N}^{k} C_{j}+\sum_{j=N}^{k} \frac{v_{j}^{2}}{R_{j} /(1+\varepsilon)+v_{j}}
$$

and hence

$$
v_{N} \geq \sum_{j=N}^{k} C_{j}+\sum_{j=N}^{k} \frac{v_{j}^{2}}{R_{j} /(1+\varepsilon)+v_{j}}
$$

Letting $k \rightarrow \infty$ and using (4.1), we have

$$
\sum^{\infty} \frac{v_{k}^{2}}{R_{k} /(1+\varepsilon)+v_{k}}<\infty
$$

This, together with conditions (3.3) and (4.5), implies that $v_{k} \rightarrow 0$ as $k \rightarrow \infty$. Hence by the Taylor formula for the function $\tilde{F}(k, v):=\left(R_{k}+v\right) H(k, v)$ at the center $v=0$ (see the computations in the proof of Theorem 4.1 and observe that (4.29) is still sufficient for the uniform convergence $o(1) \rightarrow 0$ as $v \rightarrow 0$ in (4.19)), we have

$$
\left(1-\frac{\varepsilon}{2}\right) \frac{v_{k}^{2}}{R_{k}+v_{k}}<H\left(k, v_{k}\right)<\left(1+\frac{\varepsilon}{2}\right) \frac{v_{k}^{2}}{R_{k}+v_{k}} \quad \text { for large } k
$$

and we can show similarly as in the proof of Theorem 4.1 (note that (4.29) implies that (4.23) holds in view of (4.22)) that

$$
\left(1+\frac{\varepsilon}{2}\right) \frac{v_{k}^{2}}{R_{k}+v_{k}}<\frac{v_{k}^{2}}{R_{k} /(1+\varepsilon)+v_{k}} \quad \text { for large } k
$$

Consequently, from (4.32),

$$
\Delta v_{k}+C_{k}+H\left(k, v_{k}\right)<0
$$

which according to Lemma 2.5 means that

$$
w_{k+1}+c_{k}-\frac{r_{k} w_{k}}{\Phi\left(\Phi^{-1}\left(r_{k}\right)+\Phi^{-1}\left(w_{k}\right)\right)}<0
$$

where $w_{k}=h_{k}^{-p} v_{k}+\widetilde{w}_{k}$ and $\widetilde{w}_{k}=r_{k} \Phi\left(\Delta h_{k} / h_{k}\right)$ is a solution of the Riccati equation associated with (1.7), hence $r_{k}+\tilde{w}_{k}>0$. Since $v_{k} \geq 0$ for large $k$, we have $r_{k}+w_{k}=r_{k}+h_{k}^{-p} v_{k}+\tilde{w}_{k}>0$ and nonoscillation of (1.1) follows from Lemma 2.2. 


\section{Remarks and applications}

We start this section with a discussion of the continuous counterparts of the results presented in the previous sections. In [15] and the subsequent papers [10, 16, 17], (1.2) is viewed as a perturbation of another half-linear differential equation of the same form

$$
\left(r(t) \Phi\left(x^{\prime}\right)\right)^{\prime}+\widetilde{c}(t) \Phi(x)=0
$$

and it is supposed that this equation possesses a positive solution $h$ such that $h^{\prime}(t) \neq 0$ for large $t$. Denote $G(t):=r(t) h(t) \Phi\left(h^{\prime}(t)\right)$ and consider the differential equation

$$
v^{\prime}+(c(t)-\widetilde{c}(t)) h^{p}(t)+(p-1) r^{1-q}(t) h^{-q}(t) H(t, v)=0,
$$

where

$$
H(t, v):=|v+G(t)|^{q}-q v \Phi^{-1}(G(t))-|G(t)|^{q}
$$

By a direct computation, similar to that given in the proof of Lemma 2.5, one can show that this equation has a solution defined on some interval $[T, \infty)$ if and only if the Riccati equation associated with (1.2)

$$
w^{\prime}+c(t)+(p-1) r^{1-q}(t)|w|^{q}=0
$$

has a solution on $[T, \infty)$. These solutions are related by the formula $v=h^{p}\left(w-w_{h}\right)$, where $w_{h}=$ $r \Phi\left(h^{\prime} / h\right)$. The function $H$ in (5.3) is the continuous counterpart of the function $H$ given by (2.10). We have the following estimates for the function $H(t, v)$ which are proved, for example, in $[18,19]$ (we present here these estimates in a modified form with respect to [18]):

$$
\begin{aligned}
& H(t, v) \leq \frac{q}{2}|G(t)|^{q-2} v^{2}, \quad p \geq 2, \\
& H(t, v) \geq \frac{q}{2}|G(t)|^{q-2} v^{2}, \quad p \in(1,2]
\end{aligned}
$$

for every $v \in \mathbb{R}$. Moreover (see [19]), for every $M \geq 0$, there exist constants $K_{1}=K_{1}(M)$, $K_{2}=K_{2}(M)$ such that

$$
K_{1}|G(t)|^{q-2} v^{2} \leq H(t, v) \leq K_{2}|G(t)|^{q-2} v^{2}
$$

for $|v| \leq M$ and any $p>1$. These estimates enable to approximate the function ( $p-$ 1) $r^{1-q} h^{-q} H(t, v)$ in $(5.2)$ by the function

$$
K r^{1-q} h^{-q}|G|^{q-2} v^{2}=\frac{K}{r h^{2}\left|h^{\prime}\right|^{p-2}} v^{2},
$$

where $K$ is a real constant, and after this approximation, (5.2) becomes the classical Riccati equation corresponding to a linear Sturm-Liouville differential equation. This linear equation is then used to study oscillatory properties of (1.2). 
In our paper, we follow a similar idea in the discrete case. The essential difference with respect to the continuous case is that the function $H$ given by (2.10) is substantially more complicated than its continuous counterpart (5.3); in particular, we were able to formulate inequalities for the function $H$ in Lemma 2.6 only under more restrictive assumptions than in the continuous case. This is also the reason why assumptions of (non-)oscillation criteria formulated in Section 3 are more restrictive than those of oscillation criteria for (1.2) given in $[10,16]$.

Now we comment in more detail on assumptions of Theorems 4.1 and 4.2 of the previous section. Assumption (4.1) is natural since if the sum of this series is $\infty,(1.1)$ is oscillatory by Lemma 2.3. Assumptions (3.3) and (4.2) are technical and we needed them to show that the solution $v$ of (3.6) satisfies $v_{k} \rightarrow 0$ as $k \rightarrow \infty$. Assumptions (3.3) and (4.2) can be replaced by a formally less restrictive assumption that

$$
\sum^{\infty} H(k, \alpha)=\infty
$$

for every $\alpha>0$, but it may be difficult to verify this assumption in particular cases. Concerning assumptions of Theorem 4.2, we also needed them to prove that $v_{k} \rightarrow 0+$ as $k \rightarrow \infty$.

We conclude the paper with a statement illustrating application of Theorem 4.2 of the previous section.

Theorem 5.1. Consider the perturbed Euler-type difference equation

$$
\Delta \Phi\left(\Delta x_{k}\right)+\left[\frac{\gamma_{p}}{(k+1)^{p}}+d_{k}\right] \Phi\left(x_{k+1}\right)=0, \quad \gamma_{p}:=\left(\frac{p-1}{p}\right)^{p}
$$

and suppose that

$$
\lim _{k \rightarrow \infty} d_{k}(k+1)^{p+1}=\infty
$$

and that

$$
\sum^{\infty} d_{k}(k+1)^{p-1}<\infty
$$

Then (5.9) is nonoscillatory provided

$$
\underset{k \rightarrow \infty}{\limsup } \log k \sum_{j=k}^{\infty} d_{j}(j+1)^{p-1}<\frac{1}{2}\left(\frac{p-1}{p}\right)^{p-1} .
$$

Proof. Consider the sequence $h_{k}:=k^{(p-1) / p}$ and let $\widetilde{c}_{k}:=-\Delta \Phi\left(\Delta h_{k}\right) / \Phi\left(h_{k+1}\right)$. We have

$$
\begin{aligned}
\Delta h_{k} & =(k+1)^{(p-1) / p}-k^{(p-1) / p}=k^{(p-1) / p}\left[\left(\frac{k+1}{k}\right)^{(p-1) / p}-1\right] \\
& =k^{(p-1) / p}\left[1+\frac{p-1}{p k}-\frac{p-1}{2 p^{2} k^{2}}+O\left(k^{-3}\right)-1\right] \\
& =\frac{p-1}{p} k^{-1 / p}\left[1-\frac{1}{2 p k}+O\left(k^{-2}\right)\right]
\end{aligned}
$$


as $k \rightarrow \infty$; hence

$$
\Phi\left(\Delta h_{k}\right)=\left(\frac{p-1}{p}\right)^{p-1} k^{-(p-1) / p}\left[1-\frac{p-1}{2 p k}+O\left(k^{-2}\right)\right]
$$

and using the fact that $k^{-1}=(k+1)^{-1}+O\left((k+1)^{-2}\right)$ as $k \rightarrow \infty$, we have

$$
\begin{aligned}
& \Delta \Phi\left(\Delta h_{k}\right)=\left(\frac{p-1}{p}\right)^{p-1}\{ {\left[(k+1)^{-(p-1) / p}-k^{-(p-1) / p}\right] } \\
&\left.-\frac{p-1}{2 p}\left[(k+1)^{-2+1 / p}-k^{-2+1 / p}\right]\right\}+\Delta O\left(k^{-3+1 / p}\right) \\
&=\left(\frac{p-1}{p}\right)^{p-1}\left\{(k+1)^{-(p-1) / p}\left[1-\left(\frac{k+1}{k}\right)^{(p-1) / p}\right]\right.\left.-\frac{p-1}{2 p}(k+1)^{-2+1 / p}\left[1-\left(\frac{k+1}{k}\right)^{2-1 / p}\right]\right\}+O\left((k+1)^{-3+1 / p}\right) \\
&=-\left(\frac{p-1}{p}\right)^{p}(k+1)^{-2+1 / p}+O\left((k+1)^{-3+1 / p}\right) .
\end{aligned}
$$

Consequently,

$$
\tilde{c}_{k}=-\frac{\Delta \Phi\left(\Delta h_{k}\right)}{\Phi\left(h_{k+1}\right)}=\frac{\gamma_{p}}{(k+1)^{p}}+O\left((k+1)^{-p-1}\right) .
$$

To prove that (5.9) is nonoscillatory, we apply Theorem 4.2 with

$$
c_{k}=\frac{\gamma_{p}}{(k+1)^{p}}+d_{k}
$$

and $\widetilde{c}_{k}$ given by (5.16). The term $O\left((k+1)^{-p-1}\right)$ is of the form $a_{k}(k+1)^{-p-1}$, where $a_{k}$ is a bounded sequence; so we have

$$
c_{k}-\tilde{c}_{k}=d_{k}-\frac{a_{k}}{(k+1)^{p+1}}>0
$$

because of (5.10). Condition (4.1) is also satisfied since from (5.11) we have that the series

$$
\sum^{\infty}\left(c_{k}-\widetilde{c}_{k}\right) h_{k+1}^{p}=\sum^{\infty}\left[d_{k}(k+1)^{p-1}-\frac{a_{k}}{(k+1)^{2}}\right]
$$

is convergent. 
Concerning assumptions (3.3) and (4.29),

$$
\begin{aligned}
h_{k} h_{k+1}\left(\Delta h_{k}\right)^{p-2} & =k^{(p-1) / p}(k+1)^{(p-1) / p}\left(\frac{p-1}{p}\right)^{p-2} k^{-(p-2) / p}\left(1+O\left(k^{-1}\right)\right) \\
& =\left(\frac{p-1}{p}\right)^{p-2} k\left(1+O\left(k^{-1}\right)\right)
\end{aligned}
$$

and similarly

$$
G_{k}=h_{k} \Phi\left(\Delta h_{k}\right)=\left(\frac{p-1}{p}\right)^{p-1}\left(1+O\left(k^{-1}\right)\right)
$$

as $k \rightarrow \infty$. This means that both (3.3) and (4.29) are satisfied. Moreover, by a routine computation, one finds (using the discrete l'Hospital rule; see, e.g., [20, page 29]) that

$$
\lim _{k \rightarrow \infty} \frac{\sum_{j=1}^{k-1}(1 / j)}{\log k}=1 \text {. }
$$

Now, if (5.12) holds, there exists $\varepsilon>0$ such that

$$
\limsup _{k \rightarrow \infty} \log k \sum_{j=k}^{\infty} d_{j}(j+1)^{p-1}<\frac{1}{2(1+\varepsilon)}\left(\frac{p-1}{p}\right)^{p-1}=\frac{1}{2 q(1+\varepsilon)}\left(\frac{p-1}{p}\right)^{p-2} .
$$

This means that

$$
\limsup _{k \rightarrow \infty} \log k \frac{q}{2}\left(\frac{p}{p-1}\right)^{p-2} \sum_{j=k}^{\infty}(1+\varepsilon) C_{j}<\frac{1}{4}
$$

where $C$ is given by (1.9) with $h_{k}=k^{(p-1) / p}$. Then using (5.21) and (5.23) (with $R$ given by (1.9)), we have

$$
\limsup _{k \rightarrow \infty}\left(\sum^{k-1} R_{j}^{-1}\right)\left(\sum_{j=k}^{\infty}(1+\varepsilon) C_{j}\right)<\frac{1}{4}
$$

This implies, by Lemma 2.4, that (4.30) is nonoscillatory and the statement follows from Theorem 4.2 .

Remark 5.2. (i) We conjecture that (5.9) is oscillatory (under (5.10) and (5.11)) provided

$$
\liminf _{k \rightarrow \infty} \log k \sum_{j=k}^{\infty} d_{j}(j+1)^{p-1}>\frac{1}{2}\left(\frac{p-1}{p}\right)^{p-1} .
$$

The proof of this conjecture could follow essentially the same line as that of the previous theorem, with the only difference that Theorem 4.1 instead of Theorem 4.2 is used (and, of course, (2.7) is used instead of (2.8)). However, Theorem 4.1 needs $h$ to be the recessive solution of the equation

$$
\Delta \Phi\left(\Delta x_{k}\right)+\widetilde{c}_{k} \Phi\left(x_{k+1}\right)=0
$$


with $\tilde{c}_{k}$ given by (5.16), and in contrast to the continuous case (see $[18,21-23]$ ), a suitable summation characterization of the recessive solution is not known yet (note that the results of [24] do not apply to our case); so we are not able to prove that $h_{k}=k^{(p-1) / p}$ is really the recessive solution of (5.28) with $\tilde{c}$ given by (5.16). Moreover, we conjecture that condition (4.2) in Theorem 4.1 can be replaced by the weaker condition (4.29).

(ii) A typical equation to which the previous theorem applies is the Riemann-Weber-type difference equation

$$
\Delta\left(\Phi\left(\Delta x_{k}\right)\right)+\left[\frac{\gamma_{p}}{(k+1)^{p}}+\frac{\lambda}{(k+1)^{p} \log ^{2}(k+1)}\right] \Phi\left(x_{k+1}\right)=0 .
$$

By Theorem 4.2, this equation is nonoscillatory if $\lambda<(1 / 2)((p-1) / p)^{p-1}$.

(iii) The previous statement can be viewed as a partial extension of the (non-) oscillation criterion given in [25], where it is proved, among others, that the difference equation

$$
\Delta^{2} x_{k}+\frac{1}{4 k^{2}}\left[1+\frac{1}{\log ^{2} k}+\cdots+\frac{1}{\prod_{j=1}^{n-1}\left(\log _{j} k\right)^{2}}+\frac{\lambda}{\prod_{j=1}^{n}\left(\log _{j} k\right)^{2}}\right] x_{k+1}=0
$$

where $\log _{0} k=k, \log _{j} k=\log \left(\log _{j-1} k\right), j=1, \ldots, n$, is oscillatory if $\lambda>1$ and nonoscillatory if $\lambda<1$.

\section{Acknowledgments}

The research is supported by Grant no. 201/07/0145 of the Czech Grant Agency of the Czech Republic and the Research Project no. MSM0022162409 of the Czech Ministry of Education.

\section{References}

[1] R. P. Agarwal, S. R. Grace, and D. O'Regan, Oscillation Theory of Linear, Half-Linear, Superlinear and Sublinear Dynamic Equations, Kluwer Academic, Dordrecht, The Netherlands, 2002.

[2] O. Došlý and P. Řehák, Half-Linear Differential Equations, vol. 202 of North-Holland Mathematics Studies, Elsevier, Amsterdam, The Netherlands, 2005.

[3] P. Řehák, "Hartman-wintner type lemma, oscillation, and conjugacy criteria for half-linear difference equations," Journal of Mathematical Analysis and Applications, vol. 252, no. 2, pp. 813-827, 2000.

[4] P. Řehák, "Oscillatory properties of second order half-linear difference equations," Czechoslovak Mathematical Journal, vol. 51(126), no. 2, pp. 303-321, 2001.

[5] P. Řehák, "Generalized discrete Riccati equation and oscillation of half-linear difference equations," Mathematical and Computer Modelling, vol. 34, no. 3-4, pp. 257-269, 2001.

[6] P Řehák, "Oscillation criteria for second order half-linear difference equations," Journal of Difference Equations and Applications, vol. 7, no. 4, pp. 483-505, 2001.

[7] P. Řehák, "Oscillation and nonoscillation criteria for second order linear difference equations," Fasciculi Mathematici, no. 31, pp. 71-89, 2001.

[8] R. P. Agarwal, M. Bohner, S. R. Grace, and D. O'Regan, Discrete Oscillation Theory, Hindawi, New York, NY, USA, 2005.

[9] Á. Elbert and A. Schneider, "Perturbations of the half-linear Euler differential equation," Results in Mathematics, vol. 37, no. 1-2, pp. 56-83, 2000.

[10] O. Došlý and A. Lomtatidze, "Oscillation and nonoscillation criteria for half-linear second order differential equations," Hiroshima Mathematical Journal, vol. 36, no. 2, pp. 203-219, 2006.

[11] O. Došlý and M. Ünal, "Half-linear differential equations: linearization technique and its application," Journal of Mathematical Analysis and Applications, vol. 335, no. 1, pp. 450-460, 2007. 
[12] Z. Pátková, "Hartman-Wintner type criteria for half-linear second order differential equations," Mathematica Bohemica, vol. 132, no. 3, pp. 243-256, 2007.

[13] O. Došlý and P. Řehák, "Recessive solution of half-linear second order difference equations," Journal of Difference Equations and Applications, vol. 9, no. 1, pp. 49-61, 2003.

[14] L. H. Erbe and B. G. Zhang, "Oscillation of second order linear difference equations," Chinese Journal of Mathematics, vol. 16, no. 4, pp. 239-252, 1988.

[15] O. Došlý and S. Peña, "A linearization method in oscillation theory of half-linear second-order differential equations," Journal of Inequalities and Applications, no. 5, pp. 535-545, 2005.

[16] O. Došly, "Perturbations of the half-linear Euler-Weber type differential equation," Journal of Mathematical Analysis and Applications, vol. 323, no. 1, pp. 426-440, 2006.

[17] O. Došlý and J. Řezn ková, "Oscillation and nonoscillation of perturbed half-linear Euler differential equation," Publicationes Mathematicae Debrecen, vol. 71, no. 3-4, pp. 479-488, 2007.

[18] O. Došly and Á. Elbert, "Integral characterization of the principal solution of half-linear second order differential equations," Studia Scientiarum Mathematicarum Hungarica, vol. 36, no. 3-4, pp. 455-469, 2000.

[19] O. Došlý and J. Řezníčková, "Regular half-linear second order differential equations," Archivum Mathematicum, vol. 39, no. 3, pp. 233-245, 2003.

[20] R. P. Agarwal, Difference Equations and Inequalities: Theory, Methods, and Applications, vol. 228 of Monographs and Textbooks in Pure and Applied Mathematics, Marcel Dekker, New York, NY, USA, 2nd edition, 2000.

[21] M. Cecchi, Z. Došlá, and M. Marini, "Half-linear equations and characteristic properties of the principal solution," Journal of Differential Equations, vol. 208, no. 2, pp. 494-507, 2005.

[22] M. Cecchi, Z. Došlá, and M. Marini, "Corrigendum to "Half-linear equations and characteristic properties of the principal solution"," Journal of Differential Equations, vol. 221, no. 1, pp. 272-274, 2006.

[23] M. Cecchi, Z. Došlá, and M. Marini, "Limit and integral properties of principal solutions for half-linear differential equations," Archivum Mathematicum, vol. 43, no. 1, pp. 75-86, 2007.

[24] M. Cecchi, Z. Došlá, and M. Marini, "Nonoscillatory half-linear difference equations and recessive solutions," Advances in Difference Equations, no. 2, pp. 193-204, 2005.

[25] F. Luef and G. Teschl, "On the finiteness of the number of eigenvalues of Jacobi operators below the essential spectrum," Journal of Difference Equations and Applications, vol. 10, no. 3, pp. 299-307, 2004. 